\title{
Public Health Workforce in Switzerland: are public health workers lacking?
}

\author{
Fred Paccaud · Alison Weihofen - Martina Frank
}

Published online: 25 June 2013

(C) Swiss School of Public Health 2013

As public health workers, our core mission is to develop appropriate responses to the health needs of the population. This includes not only the prevention of diseases and the promotion and protection of health, but also the organization of health services and their evaluation. We want to ensure a fair distribution of public health benefits across the population (WHO 2006).

These are ambitious, but achievable aims. A first step in this direction and a condition for success is to organize the transfer of knowledge from the scientific research realm to best practice interventions in the workplace. Therefore, we believe that the health of a population can be greatly increased by a qualified workforce specifically trained in public health sciences (Beaglehole 2003). A public health education can provide knowledgeable, prepared practitioners at all levels, who can achieve improved health outcomes and contribute to large-scale changes in the overall health of our societies.

Currently, there is no shortage of discussion on the public health workforce and its training needs, especially among public health practitioners working in the public or

F. Paccaud · A. Weihofen $(\square)$

Swiss School of Public Health, Seilergraben 49,

8001 Zürich, Switzerland

e-mail: aweihofen@ssphplus.ch

URL: http://www.ssphplus.ch

F. Paccaud

e-mail: fpaccaud@ssphplus.ch

URL: http://www.ssphplus.ch

M. Frank

KEK-CDC Consultants, Universitätstrasse 69,

8006 Zürich, Switzerland

e-mail: frank@kek.ch

URL: http://www.kek.ch semi-public sectors, as well as in private companies. A report by the WHO (2006) states that our global workforce capacity (size, training, and competencies) is currently misaligned with the health needs of the population. Bjegovic-Mikanovic et al. assert in their article "Education and training of public health professionals in the European Region: variation and convergence" (2013, this issue) that public health education in Europe is vastly insufficient to prepare workers for the "real world" of changing health risks, increasing health disparities and newly developing health technologies, among other pressing challenges. This body of research suggests a critical gap exists between actual public health needs and the extent to which public health workers are trained in basic and continuing public health education.

Yet, if we are to better understand the needs of the workers, and better prepare our educational institutions to meet these needs, we need a more cohesive understanding of where public health graduates are going when they enter the workforce, and what they are doing.

There have been several attempts to map professional engagements and educational backgrounds of the public health workforce (Frank et al. 2013, http://www.ssphplus. ch/downloads). In order to better assess our domestic workforce, the Swiss School of Public Health (SSPH+) recently conducted a census and created a profile of those currently employed in public health positions (Frank et al. 2013). We found that overall, respondents were well-educated (2/3 had at least a Bologna masters degree). However, $69 \%$ of respondents in the workforce had no specific public health degree, suggesting that current workers, at all levels, could benefit from further training in specific public health skills. This finding is supported by our data showing "public health sciences" as one of the top-reported competency needs by workers. 
In addition to the lack of basic public health training, a related challenge that emerged from the recent SSPH+ study was the definition of "public health worker". Delimiting our own workforce proved challenging. The definition of public health is quite broad, and there is a wide scope of possible employers/job titles in our listed public health sectors: educational/research, government, non-governmental organizations, professional associations, hospitals, health insurances, consulting, international organizations and pharmaceutical/biotech industry. Within this highly diverse population of workers, there are many topic areas: maternal and child health, obesity, regulation, legislation, HIV/AIDS, sun protection, international rights, sexual health, arthritis, aging, natural disaster preparation, among many others. Riddout et al. (2002) and Tilson and Gebbie (2004) echo the challenges in defining the workforce, citing the variety of occupational groups in public health, the lack of professional category boundaries, the absence of required professional credentialing, and the majority of workers who lack formal public health training.

If we want to make changes in the population's health, we need a workforce specifically trained in public health skills and knowledge to carry out the challenging tasks the field of public health presents. Switzerland should also seek to create a more cohesive public health workforce identity and increase its visibility. The lessons learned from the
Swiss census have implications for the wider European public health workforce. A systematic and regular monitoring of the public health workforce is essential to increase our understanding of its needs and related strategies to training the workforce. Only with increased knowledge can we better understand the educational needs of current public health workers making crucial decisions that affect our population's health, as well as assess the adequacy of the workforce size needed to meet future public health challenges.

\section{References}

Beaglehole R, Dal Poz MR (2003) Public health workforce: challenges and policy issues Human. Resour Health 1(1):4

Frank M, Weihofen A, Duetz Schmucki M, Nocera S, Paccaud F (2013) Public Health Workforce in Switzerland: a National Census. Swiss School of Public Health Zurich Switzerland, Federal Office of Public Health Bern, Switzerland

Riddout L, Gadiel D, Cook K, Wise M (2002) Planning framework for the Public Health Workforce. Discussion paper. National Public Health Partnership, Melbourne

Tilson H, Gebbie KM (2004) The public health workforce. Annu Rev Public Health 25:341-356

World Health Organization (2006) Working together for health: World Health Report 2006. Switzerland, Geneva 